\title{
Rapid conversion of replicating and integrating Saccharomyces cerevisiae plasmid vectors via Cre recombinase
}

\author{
Daniel P. Nickerson ${ }^{1 *}$, Monique A. Quinn ${ }^{1}$, and Joshua M. Milnes ${ }^{2,3}$ \\ ${ }^{1}$ Department of Biology, California State University, San Bernardino, CA 92407 \\ ${ }^{2}$ Department of Biochemistry, University of Washington School of Medicine, Seattle, WA 98195- \\ 3750 \\ ${ }^{3}$ Present address: Washington State Department of Agriculture - Plant Protection Division, \\ Yakima, WA 98902 \\ *Corresponding author: daniel.nickerson@csusb.edu
}

Key words: Cre recombinase, homologous recombination, plasmid, shuttle vector, overexpression, molecular cloning, genetic engineering, yeast

Running title: Remodeling yeast vector replication loci 


\section{$1 \quad$ ABSTRACT}

2 Plasmid shuttle vectors capable of replication in both Saccharomyces cerevisiae and Escherichia

3 coli and optimized for controlled modification in vitro and in vivo are a key resource supporting

4 yeast as a premier system for genetics research and synthetic biology. We have engineered a

5 series of yeast shuttle vectors optimized for efficient insertion, removal and substitution of

6 plasmid yeast replication loci, allowing generation of a complete set of integrating, low copy

7 and high copy plasmids via predictable operations as an alternative to traditional subcloning.

8 We demonstrate the utility of this system through modification of replication loci via Cre

9 recombinase, both in vitro and in vivo, and restriction endonuclease treatments. 
Nickerson et al. p3

\section{INTRODUCTION}

2 Yeast (S. cerevisiae) has long served as a premier experimental system for exploring eukaryotic

3 genetics and cell biology (Botstein and Fink 2011) and has more recently emerged as a

4 preferred system in synthetic biology (Lee et al. 2015). A key component of the yeast genetic

5 toolkit is the availability of shuttle vectors, plasmids capable of replication and selection when

6 introduced into either a yeast or E. coli cell (Sikorski and Hieter 1989; Da Silva and Srikrishnan

7 2012). In their original design, yeast shuttle vectors would typically be modified in vitro and

8 subsequently propagated in bacterial cells to generate a desired gene construct that could be

9 later introduced into yeast to study eukaryotic cell biology. In current practice the classical

10 bacteria-then-yeast workflow is often turned on its head by gene synthesis techniques that

11 capitalize upon the readiness of yeast to piece together compatible DNA fragments via

12 homologous recombination (Gibson 2009), so initial gene synthesis and molecular cloning steps

13 often start inside the yeast cell.

Yeast shuttle vectors vary in their selectable markers, typically genes for auxotrophic

15 rescue or antibiotic resistance, and in the presence or absence of a yeast replication locus.

16 Yeast integrating plasmids lack an independent replication locus and must be incorporated into

17 a chromosome in order to replicate. Low copy, centromeric plasmids contain an autonomous

18 replicating sequence (ARS) to initiate DNA replication and a centromere (CEN) to support

19 plasmid inheritance during cell division. High copy, episomal vectors carry the $2 \mu$ circle

20 replicating origin. Low and high copy plasmids offer convenience for gene expression studies

21 and functional analyses, but both varieties of replicating plasmids have demonstrated instability

22 in their copy numbers (Futcher and Carbon 1986; Mead et al. 1986; Resnick et al. 1990). When 
1 considering the possible toxicity of plasmid-encoded gene or combination of genes, or even the

2 energetic cost of maintaining the plasmid itself (Mead et al. 1986), unstable copy numbers of

3 centromeric and $2 \mu$ plasmids can present clear obstacles to experimental execution and

4 interpretation. To ensure even copy number and consistent level of gene expression, some

5 plasmid-encoded genes must be integrated into a chromosome, but such necessity is often

6 realized after experimentation with expression from replicating plasmids. Ability to switch

7 between low copy, high copy and integrating shuttle vector strategies is a key feature of the

8 yeast genetics toolkit.

Previous efforts in improving resources for yeast shuttle vectors have focused heavily on engineering sequences flanking the yeast selectable marker loci to offer convenient removal

11 and remodeling (Gueldener et al. 2002; Carter and Delneri 2010; Fang et al. 2011; Chee and

12 Haase 2012; Agaphonov and Alexandrov 2014; Siddiqui et al. 2014; Jensen et al. 2014), either in

13 the plasmid or after chromosomal integration. By comparison, the replication loci of the shuttle

14 vectors have been neglected. We believe a similar level of utility is achieved by engineering the

15 yeast replication loci of shuttle vectors to be removed and remodeled in analogous manner.

We have modified the popular family of pRS shuttle vectors (Sikorski and Hieter 1989;

17 Christianson et al. 1992) plasmid shuttle vectors to include yeast replication loci flanked by LoxP

18 sites or triplicate endonuclease cut sites. We demonstrate the utility and flexibility of this

19 system through in vivo and in vitro remodeling of the yeast replication loci and the rapid

20 generation of a complete suite of integrating, low copy and high copy plasmids to support

21 functional analysis in yeast. 
Nickerson et al. p5

\section{$1 \quad$ RESULTS \& DISCUSSION}

pDN5xx \& pDN6xx vector series

3 While several families of yeast shuttle vectors have been deployed over nearly four decades

4 (reviewed in Da Silva and Srikrishnan 2012), availability of the pRS series of vectors (Sikorski and

Hieter 1989; Christianson et al. 1992) was a landmark in yeast genetics; the plasmids were

rapidly adopted by the field and their use remains ubiquitous due to several useful features

(Figure $1 \mathrm{~A}$ ): i/ yeast auxotrophic selection markers (HIS3, TRP1, LEU2 or URA3) are compatible

with engineered auxotrophies in many of the most commonly used yeast laboratory strains

(Sherman 2002); ii/ plasmid selection in bacteria via ampicillin resistance; iii/ bacterial origins of

replication that produce high copy number and high plasmid yield upon extraction from

11 bacteria; iv/ a large polylinker or multiple cloning site (MCS) with a selection of unique

13 promoters flanking the MCS to permit in vitro RNA transcription; and vi/ a $\beta$-galactosidase

14 coding region overlapping the MCS to permit colorimetric screening of bacterial colonies for

15 successful integration of DNA insert.

In designing the pDN500 and -600 series (Figure $1 \mathrm{~A}$ ), we honored the original

17 numbering convention of the pRS series in which the second numeral indicates the yeast

18 replication locus (' 0 ' for none, ' 1 ' for centromeric, and ' 2 ' for $2 \mu$ ) and the third indicates the

19 yeast selectable marker (' 3 ' for HIS3, ' 4 ' for TRP1, ' 5 ' for LEU2, and '6' for URA3). In the

20 pDN500-series, yeast replication loci are flanked by pairs of restriction endonuclease cut sites,

21 enabling targeted in vitro removal of either CEN or $2 \mu$ loci. The trio of Aatll, Aatll and Sphl were

22 selected because these enzymes are commonly used for laboratory cloning and lack cut sites 
1 elsewhere in the pRS and pDN family of vectors, excepting that Avrll cuts in the HIS3 locus,

2 consistent with characterization of the pRS vectors by (Chee and Haase 2012). Three pairs of

3 flanking endonuclease cut sites were included to maximize the likelihood that at least one pair

4 of cut sites should remain available to modify the replication locus in case further DNA inserts

5 might contain cut sites for one or two of these enzymes. Importantly, removal of the replication

6 locus by flanking restriction digest supports either elimination of the locus by plasmid re-

7 circularization or substitution of an alternative replication locus.

The pDN61x and -62x series of vectors allow remodeling of yeast replication loci via a

pair of parallel, flanking LoxP sequences, offering options to remodel the plasmid by Cre

recombinase activity either in vivo or in vitro. The Cre-Lox recombination strategy offers the

11 further advantage that the option to remodel a yeast replication locus will persist regardless of

12 what further DNA sequences might be inserted into the plasmid, provided no additional LoxP

13 sites are included. pDN600-series vectors also include a pair of flanking Aatll cut sites on either

14 side of the replication loci, so remodeling options available to the pDN500-series also apply.

17 We demonstrated working procedures for remodeling replication loci in the pDN600-series

18 using plasmid pJM1 (Figure $2 \mathrm{~A}$ ), generated by incorporating a gene (GFP-CPS) encoding GFP-

19 tagged transmembrane endosomal cargo reporter carboxypeptidase $S$ into the centromeric,

20 uracil-selected vector pDN616. Chromosomal integration is particularly useful for plasmids

21 encoding fluorescent reporters, since uneven expression across a population of cells can result

22 in a range of signal intensities and possible phenotypes. We converted pJM1 into a replication- 
1 deficient, integrating plasmid (pJM3) via Cre recombinase activity toward the LoxP-flanked CEN/ARSH4 locus. We passaged pJM1 through E. coli strain N2114Sm (Seifert et al. 1986) that stably expresses Cre. Plasmid DNA was extracted from ampicillin-selected N2114Sm colonies and used to transform E. coli strain TOP10F' for the dual purpose of filtering the polyclonal plasmid population immediately derived from N2114Sm and achieving a higher yield of plasmid

DNA. Restriction analysis of the resulting pJM3 candidates revealed that neither candidate retained the Aatll cut sites that flank the CEN/ARSH4 locus in pJM1 (Figure $2 \mathrm{~B}$ ). Digestion with a combination of EcoRV and Pvul further confirmed that a pJM1 restriction product containing the CEN/ARSH4 locus (1834 bp) was absent in pJM3 candidates, but that the restriction fragment at $1250 \mathrm{bp}$ in $\mathrm{pJM} 3$ had doubled in relative intensity, indicating the presence of two

11 fragments, consistent with the $1834 \mathrm{bp}$ fragment being reduced to $1250 \mathrm{bp}$ by removal of the CEN/ARSH4 sequence by successful Cre-Lox recombination. We confirmed competence of pJM3 as an integrating plasmid by cutting at the unique

14 Sacll recognition site in the PRC1 promoter sequence driving expression of GFP-CPS, producing

15 linearized pJM3 with end sequences to direct integration via homologous recombination into the chromosomal PRC1 promoter. We transformed linearized pJM3 into wild type yeast and a

17 vps $4 \Delta$ mutant strain that suffers an endosome maturation defect preventing formation of

18 luminal vesicles (Babst et al. 1997), confirmed integration in colonies selected on media lacking

19 uracil, and cultured cells under non-selective conditions prior to pulse-labeling with a

20 fluorescent endocytic membrane dye, FM4-64 (Vida and Emr 1995) to stain the perimeter of

21 the yeast vacuole. GFP-CPS transits via the biosynthetic pathway from Golgi to endosome

22 where it is sorted into luminal endosomal vesicles (Odorizzi et al. 1998). Luminal vesicles and 
Nickerson et al. p8

1 GFP-CPS cargo are delivered to the vacuole lumen when endosomes fuse with the vacuole, so

2 in wild type cells GFP signal appeared inside the FM4-64-stained vacuole membrane (Figure 2

3 C). Loss of the gene VPS4 (vps4D) disrupts the ability of endosomes to invaginate and form

4 luminal vesicles, so GFP-CPS remains at the outer membrane of endosomes and is delivered

5 instead to the outer membrane of the vacuole, co-localizing with FM4-64 at the vacuole membrane and at perivacuolar endosomal compartments (Figure $2 \mathrm{C}$ ). These observations are consistent with previous studies (Odorizzi et al. 1998) and confirm performance of the pDN600 series in converting to integration-competent vectors.

We further examined whether Cre would remove LoxP-flanked $2 \mu$ replication loci. We passaged the high copy vector pDN624 through Cre-expressing bacteria via the same procedure

11 described above. Restriction analysis using endonuclease EcoRI revealed that all Cre-treated

12 candidates had been reduced in length $\sim 1400 \mathrm{bp}$ compared to untreated pDN624 (Figure 3 A),

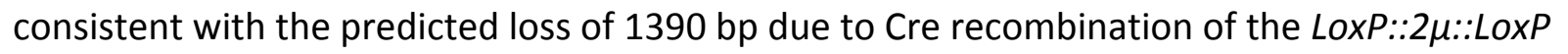
cassette. Purified Cre enzyme is readily available from commercial suppliers, so we also examined whether LoxP-flanked yeast replication loci could be removed in vitro. Cre-treatment of high copy plasmids pDN624 and pDN626 for only thirty minutes resulted in modification of a

17 substantial subpopulation of the plasmids (Figure 3 B). Treated samples possessed bands

18 representing unmodified pDN624 and pDN626 as well as an additional band 1400 bp shorter

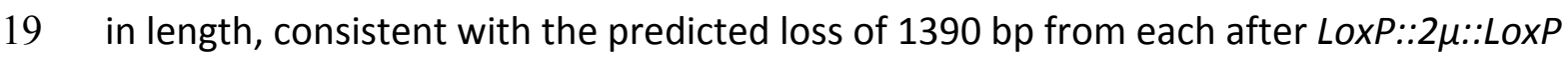

20 recombination. Users of the pDN600 series therefore have the option to conduct Cre-mediated 21 remodeling of the yeast replication locus either in vivo or in vitro. 
Nickerson et al. p9

Generation of integrating plasmids by restriction digest to excise plasmid replication loci

2 We explored the plasticity of the pDN500-series by generating a full suite of replicating and

3 integrating plasmid vectors expressing a mutant allele (L291A, L292A-or 'LALA') of the SNARE

4 disassembly adaptor protein Sec17 (Schwartz et al. 2017). We generated a sec17 ${ }^{L A L A}$ PCR

5 product with ends homologous to the ends of Sacl-digested pDN516, inserting $\sec 17^{\text {LALA }}$ into

6 pDN516 via co-transformation into yeast cells for plasmid gap repair by homologous

7 recombination (Figure 4 A). The resulting low copy, centromeric plasmid (pDN366) was

8 subsequently converted to a non-replicating, integrating plasmid by digesting pDN366 with

9 Aatll and re-circularizing with T4 ligase to remove the CEN/ARSH4 locus, resulting in pDN370. In

10 order to generate a high copy plasmid to overexpress $\sec 17^{\operatorname{LALA}}, \mathrm{pDN} 370$ was again cut with

11 Aatll to make linear ends available for insertion of the $2 \mu$ locus. We examined efficiency of $2 \mu$

12 locus insertion into pDN370 via Gibson assembly (Gibson et al. 2009), which like gap repair

13 cloning in yeast also relies upon overlapping homologous sequences at the ends of vector and

14 insert, resulting in pDN369.

All three versions of the $\sec 17^{\text {LALA }}$ plasmid suite produce a $6185 \mathrm{bp}$ band upon Aatll

16 digestion (Figure $4 \mathrm{~B}$ ), representing the $\sec 17^{\mathrm{LALA}}$ gene insert and the remainder of the common

17 plasmid backbone that lacks the yeast replication locus. pDN366 and pDN369 also produce Aatll

18 restriction fragments at 563 or 1369 bp, representing CEN/ARSH4 or $2 \mu$ loci, respectively.

19 Candidate plasmids examined after recircularization of pDN366 to produce pDN370 all lack the

$20563 \mathrm{bp}$ CEN/ARSH4 locus (Figure $4 \mathrm{C}$ ), which was expected given the known robustness of the

21 recircularization technique. Screening ten candidate plasmids for high copy pDN369 generated

22 by Gibson assembly also revealed a high degree of successful $2 \mu$ locus insertion (80\%). 
The workflow described in Figure 4 works equivalently if the starting vector is high copy

$2(2 \mu)$ instead of low copy (CEN/ARSH4); such an alternative workflow was used to generate

3 LUCID family cargo transport reporter plasmids (Nickerson et al. 2012; Nickerson and Merz

4 2015) in which multiple genes expressing chimeric reporter enzymes were inserted at the MCS,

5 so the ability to remodel the replication loci of the plasmids was far preferable to subcloning

6 large, multi-component gene inserts.

7 A further convenience of a standard family of yeast shuttle vectors with ability to

8 remodel the replication loci is the limited number of needed reagents for a research lab to

9 stock. Indeed, remodeling operations demonstrated in this study could be performed using the

10 enzymes Aatll, Cre, and T4 ligase, plus frozen stocks of CEN/ARSH4 and $2 \mu$ PCR products ready

11 to insert.

12 Future improvements to shuttle vector systems could merge the benefits of targeted

13 removal or remodeling of both the yeast replication loci and the yeast selectable markers. The

14 use of Cre-Lox recombination in the pDN600-series presents an incompatibility with the

15 commonly used Cre-Lox removal of selectable marker loci, but there are several alternative

16 recombinase enzymes and recognition sequences available to incorporate. 
Nickerson et al. p11

\section{MATERIALS \& METHODS}

$$
\text { Media and reagents }
$$

Standard methods were used for culture and storage of yeast and bacteria (Guthrie and Fink 2002). All media and reagents were purchased from Sigma-Aldrich (Saint Louis, MO) or Thermo

Fisher (Waltham, MA), unless otherwise specified. All enzymes were purchased from New England Biolabs (Ipswich, MA) unless otherwise specified. High fidelity KOD Hot Start polymerase was purchased from Novagen/EMD Millipore (Darmstadt, Germany). DNA restriction digests, T4 DNA ligase reactions and PCR reactions were all performed according to manufacturer instructions. Oligonucleotide synthesis was performed by Integrated DNA Technologies (Corralville, IA). Hyperladder I (Bioline) was used as linear DNA size standard.

\section{DNA manipulations and reagents}

Strains and plasmids used in this study are described in Table I. Oligonucleotides used in this study are described in Table II. In constructing the pDN51x or pDN52x plasmid series, PCR primer pairs (DN652p \& DN653p or DN1016p \& DN1017p) were designed to amplify either the CEN/ARSH4 locus or the high copy $2 \mu$ locus from pRS415 or pRS425 templates, respectively, while incorporating Aatll, AvrlI, and Sphl restriction sites ('3X') flanking the loci. To construct the pDN61x plasmid series, PCR primer pairs (DN648p \& DN649p or MQ1p \& MQ2p) were designed to amplify CEN/ARSH4 or $2 \mu$ loci from pRS415 or pRS425 templates, respectively, while incorporating parallel LoxP sequences flanking the loci. After successful high-fidelity PCR amplification, template plasmid DNA was degraded by treatment with Dpnl restriction enzyme prior to precipitation of PCR product and resuspension in 0.1M LiOAc $2 \mathrm{mM}$ Tris $\mathrm{pH}$ 7.9. The 


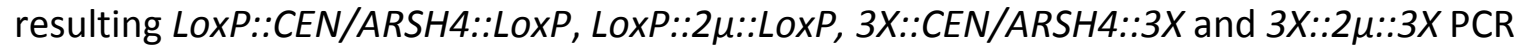

products were co-transformed into yeast (S. cerevisiae) with Aatll-digested pRS403, pRS404,

pRS405 or pRS406 linearized vectors using a lithium acetate-based protocol described below.

Homologous recombination of the replication loci and linearized integrating vectors yielded

new, low- and high-copy replicating vectors. pDN5xx- and pDN6xx-series plasmid candidates

were screened and confirmed by restriction digest, DNA sequencing, competence for Cre-

mediated recombination, and ability to support yeast and bacterial colony growth after

8 transformation and plating onto selective media.

plasmid vectors by homologous recombination with compatible DNA insert was adapted from

11 (Gietz et al. 1992). Cells were shaken overnight in YPD media at $30^{\circ} \mathrm{C}$. Saturated cultures were

12 diluted to $\mathrm{OD}_{600} \sim 0.1$ and shaken under identical conditions until cells reached log phase

13 density $\left(O D_{600}=0.4-0.6\right)$. Cells were collected by low speed centrifugation and rinsed in $0.1 \mathrm{M}$

14 LiOAc $2 \mathrm{mM}$ Tris $\mathrm{pH}$ 7.9. Cell pellets were resuspended in $50 \mu \mathrm{L} 0.1 \mathrm{M}$ LiOAc $2 \mathrm{mM}$ Tris $\mathrm{pH} 7.9$

15 containing either resuspended PCR product ('insert') or no PCR product as a negative control.

16 Cell suspensions were further supplemented with 10-50 $\mu \mathrm{g}$ boiled salmon sperm DNA and

17 approximately $25 \mathrm{ng}$ linearized plasmid vector before dilution with $700 \mu \mathrm{L} 40 \%$ PEG (w/v) in

$18 \quad 0.1 \mathrm{M}$ LiOAc $2 \mathrm{mM}$ Tris $\mathrm{pH}$ 7.9. Cell suspensions were vortexed $10-20$ seconds and incubated at

$1930^{\circ} \mathrm{C}$ for $15-30$ minutes. Cell suspensions were supplemented with $5 \%(v / v)$ DMSO and vortexed

20 another 10 seconds prior to a heat shock incubation at $42^{\circ} \mathrm{C}$ for 30 minutes. Cells were

21 collected by low speed centrifugation and resuspended in 0.1M LiOAc $2 \mathrm{mM}$ Tris $\mathrm{pH} 7.9$ prior to

22 spreading on selective agar media. 

protocol (Rose et al. 1990) of glass bead cell lysis, phenol-chloroform extraction and ethanol precipitation of the aqueous phase to yield genomic and plasmid DNA. Plasmids were separated

4 from genomic DNA by electroporation of $E$. coli and plating of cells to LB agar media with

5 ampicillin $(100 \mu \mathrm{g} / \mathrm{mL})$. Plasmids were recovered from bacteria using a QIAgen plasmid miniprep kit (Qiagen, Valencia, CA). DNA sequencing reactions of replication loci using flanking primers DN661p or DN837p was performed by Genewiz (South Plainfield, NJ). Sequencing manufacturer's instructions (New England Biolabs). alignments were performed using SnapGene software (GSL Biotech, San Diego, CA). Cre-mediated removal of LoxP-flanked replication loci from pDN61x- and pDN62x-series vectors was accomplished in vivo by chemical transformation of an $E$. coli strain expressing Cre recombinase, N2114Sm (Seifert et al. 1986). Ampicillin-selected colonies were picked and

14 grown in LB media supplemented with ampicillin $(50 \mu \mathrm{g} / \mathrm{mL})$ before plasmid extraction via QIAgen plasmid miniprep. Plasmid yields from N2114Sm host strain are low, so purified plasmid candidates were further passaged through TOP10F' E. coli (Invitrogen, Carlsbad, CA) via

17 chemical transformation and QIAgen plasmid miniprep extraction. accomplished in vitro treating 250 ng plasmid with purified Cre recombinase enzyme (New

20 England Biolabs) in $50 \mu \mathrm{L}$ reaction per manufacturer instructions, incubating at $37^{\circ} \mathrm{C}$ for 30

21 minutes. Resulting polyclonal Cre recombinase reactions were heat inactivated and plasmid 
Nickerson et al. p14

1 DNA was precipitated twice using ethanol prior to restriction digest and electrophoretic

2 analysis.

Plasmid pJM1 was constructed via PCR amplification of the PRC1 promoter-driven GFP-

DN693p, creating a PCR product with ends overlapping the ends of Pvull-cut vector pDN616.

site (MCS), removing the MCS entirely, thus eliminating many redundant endonuclease cut

sites. PCR product and Pvull-cut pDN616 were co-transformed into yeast to perform

homologous recombination plasmid repair as described above. Transformants were selected on

agar media lacking uracil to select circularized plasmids. pJM1 was modified to generate pJM3

11 by removal of the LoxP-flanked CEN/ARSH4 locus by passaging $\mathrm{pJM} 1$ through Cre-expressing

12 strain N2114Sm as described above. pJM3 was linearized for chromosomal integration by Sacll

13 digestion of the unique cut site in the $P R C 1$ promoter sequence, generating linear ends capable

14 of mediating homologous recombination at the PCR1 chromosomal locus. Strains SEY6210 and

15 MBY3 were both transformed using Sacll-cut pJM3 using the high-efficiency protocol described

16 above.

20 primers DN927p and DN928p included 35 and $34 \mathrm{bp}$, respectively, sequence overlapping the

21 linear ends of Sacl-digested pDN516, allowing insertion of $\sec 17^{\text {LALA }}$ PCR product into the

22 pDN516 MCS via homologous recombination plasmid repair, resulting in pDN366. Aatll digest of 
1 pDN366 linearized the plasmid and removed the CEN/ARSH4 locus. Compatible Aatll overhangs

2 were ligated together using T4 ligase, omitting the CEN/ARSH4 locus and resulting in pDN370.

3 Insertion of $2 \mu$ replication locus PCR product into Aatll-digested pDN370 via Gibson cloning was

4 performed essentially as described (Gibson et al. 2009), resulting in pDN369. PCR primers

5 DN652p and DN653p used to amplify the $2 \mu$ locus include $30 \mathrm{bp}$ overhangs homologous to ends

6 of Aatll-digested pDN5xx vectors, making them suitable to mediate both homologous

7 recombination plasmid repair in yeast and in vitro overlap extension via Gibson cloning.

\section{8}

\section{Imaging}

Pulse-chase labeling of log phase yeast with vacuolar fluorescent dye FM4-64 and fluorescence microscopy imaging were performed as described (Nickerson et al. 2012), except that cells were grown in non-selective YPD prior to labeling and imaging. DNA in agarose gels was stained using ethidium bromide and visualized using ultraviolet light in a BioRad Chemi-doc system with digital camera and Quantity One imaging software (BioRad, Hercules, CA). All gel images were exported from Quantity One as .TIFF images, except Figure 2 panel B, which was printed to photographic paper and later scanned in .TIFF format using an Epson flatbed scanner. Images were cropped using Adobe Photoshop CS6 (Adobe, San Jose, CA). Fluorescence microscopy images were overlaid using ImageJ (NIH, https://imagej.nih.gov/ij/). Images were arranged as annotated figures using Canvas Draw 4 vector graphics software (Canvas GFX, Boston, MA). 
2 Vector sequence files and maps may be downloaded in SnapGene format (.dna) as

3 supplementary files. Plasmid vectors in the pDN51x, pDN52x, pDN61x, and pDN62x vector

4 series will be deposited at AddGene (Cambridge, MA). Direct reagent requests to the Nickerson

5 Lab should please provide a self-addressed, stamped envelope, mailed to 'Attn: Nickerson,

65500 University Pkwy, CSUSB Biology Dept, Rm BI-302, San Bernardino, CA 92407-2318.'

\section{AUTHOR CONTRIBUTIONS}

10 DPN conceived of the project. DPN and MAQ conceived and designed experiments. DPN, JMM

11 and MAQ performed experiments and analyzed results. DPN wrote the manuscript with editing 12 contributions from MAQ and JMM.

ACKNOWLEDGMENTS

16 This work was supported by grants from the CSUSB Office of Student Research to MAQ and

17 DPN, charitable donations to the Nickerson Lab Research Fund (CSUSB), and NIH/NIGMS RO1

18 GM077349 to AJM (University of Washington). 


\section{TABLES}

Table I. Plasmids and strains used in this study.

Name

Genotype/Description

\section{Plasmids}

pDN613
pDN614
pDN615
pDN616
pDN623
pDN624
pDN625
pDN626
pDN513
pDN514
pDN515
pDN516
pDN523
pDN524
pDN525
pDN526
pJM1
pJM3
pDN314
pDN366
pDN369
pDN370
pRS403
pRS404
pRS405
pRS406
pRS415
pRS425
pG045

Amp ${ }^{\mathrm{R}}$ HIS3 LoxP::CEN/ARSH4::LOXP

Amp $^{\mathrm{R}}$ TRP1 LOXP::CEN/ARSH4::LOXP

Amp $^{\mathrm{R}}$ LEU2 LOXP::CEN/ARSH4::LOXP

Amp $^{\mathrm{R}}$ URA3 LOXP::CEN/ARSH4::LOXP

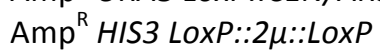

Amp $^{\mathrm{R}}$ TRP1 LoXP::2 $\mu::$ LoXP

Amp $^{\mathrm{R}}$ LEU2 LoXP::2 $\mu::$ LoXP

Amp $^{\mathrm{R}}$ URA3 LoXP::2 $\mu::$ LOXP

Amp $^{\mathrm{R}}$ HIS3 $3 X:: C E N / A R S H 4:: 3 X$

Amp $^{\mathrm{R}}$ TRP1 3X::CEN/ARSH4::3X

Amp $^{\mathrm{R}}$ LEU2 3X::CEN/ARSH4::3X

Amp $^{\mathrm{R}}$ URA3 3X::CEN/ARSH4::3X

Amp $^{\mathrm{R}}$ HIS3 $3 X:: 2 \mu:: 3 X$

Amp $^{\mathrm{R}}$ TRP1 $3 X:: 2 \mu:: 3 X$

$\operatorname{Amp}^{\mathrm{R}}$ LEU2 $3 X:: 2 \mu:: 3 X$

Amp $^{\mathrm{R}}$ URA3 $3 X:: 2 \mu:: 3 X$

Amp ${ }^{\mathrm{R}}$ URA3 LOXP::CEN/ARSH4::LOXP PRC1pr::GFP-CPS1

Amp $^{\mathrm{R}}$ URA3 LOXP PRC1pr::GFP-CPS1

Amp $^{\mathrm{R}}$ URA3 $3 X:: 2 \mu:: 3 X$ SEC17

$\mathrm{Amp}^{\mathrm{R}}$ URA3 3X::CEN/ARSH4::3X sec17 ${ }^{\text {L291A/L292A }}$

Amp $^{\mathrm{R}}$ URA3 $3 X:: 2 \mu:: 3 X \sec 17^{L 291 A / L 292 A}$

Amp $^{R}$ URA3 $\sec 17^{L 291 A / L 292 A}$

$\mathrm{Amp}^{\mathrm{R}}$ HIS3

$\mathrm{Amp}^{\mathrm{R}}$ TRP1

$\mathrm{Amp}^{\mathrm{R}}$ LEU2

$\mathrm{Amp}^{\mathrm{R}} \cup R A 3$

$\mathrm{Amp}^{\mathrm{R}}$ LEU2 CEN/ARSH4

$\operatorname{Amp}^{\mathrm{R}}$ LEU2 $2 \mu$

Amp $^{\mathrm{R}}$ URA3 $2 \mu$ PRC1pr::GFP-CPS1

E. coli
Source/Reference

This study

(Shideler et al. 2015)

(Paulsel et al. 2013)

(Nickerson et al. 2012)

This study

This study

This study

This study

This study

This study

This study

This study

This study

(Lobingier et al. 2014)

This study

(Nickerson et al. 2012)

This study

This study

(Lobingier et al. 2014)

This study

(Schwartz et al. 2017)

This study

(Sikorski and Hieter 1989)

(Sikorski and Hieter 1989)

(Sikorski and Hieter 1989)

(Sikorski and Hieter 1989)

(Sikorski and Hieter 1989)

(Christianson et al. 1992)

(Odorizzi et al. 1998)

Invitrogen

(Seifert et al. 1986)

(Robinson et al. 1988)

(Babst et al. 1997)

This study

This study 
Table II. Primer sequences used in this study. Sequences to mediate homologous recombination shown in bold. LoxP sequences shown in italics. Restriction site sequences shown as underlined. PCR annealing sequences shown in lowercase.

DN648p: Rev, PCR amplify CEN/ARSH4 sequence, incorporate LoxP sequence and Aatll restriction site, mediate repair of Aatll-digested $p R S 40 x$ vector.

5'GGTTAATGTCATGATAATAATGGTTTCTTAATAACTTCGTATAGCATACATTATACGAAGTTATGACGT Cggacggatcgcttgcctg-3'

DN649p: Fwd, PCR amplify CEN/ARSH4 sequence, incorporate LoxP sequence and Aatll restriction site, mediate repair of Aatll-digested $\mathrm{pRS40x}$ vector.

5'CGCGCACATTTCCCCGAAAAGTGCCACCTATAACTTCGTATAATGTATGCTATACGAAGTTATTGACGT Ccccgaaaagtgccacctg-3'

DN652p: Fwd, PCR amplify CEN/ARSH4 sequence, incorporate triplicate restriction sites, mediate repair of Aatll-digested pRS40x or pDN50x vectors.

5'CACATTTCCCCGAAAAGTGCCACCTGACGTCCTAGGCATGCggtccttttcatcacgtgc-3'

DN653p: Rev, PCR amplify CEN/ARSH4 sequence, incorporate triplicate restriction sites, mediate repair of Aatll-digested pRS40x or pDN50x vectors.

5'ATGTCATGATAATAATGGTTTCTTAGACGTCCTAGGCATGCgataataatggtttcttag-3'

DN1016p: Fwd, PCR amplify $2 \mu$ sequence, incorporate triplicate restriction sites, mediate repair of Aatll-digested pRS40x or pDN50x vectors.

5'CACATTTCCCCGAAAAGTGCCACCTGACGTCCTAGGCATGCaacgaagcatctgtgcttcatt-3'

DN1017p: Rev, PCR amplify $2 \mu$ sequence, incorporate triplicate restriction sites, mediate repair of Aatll-digested $p R S 40 x$ or pDN50x vectors.

5'ATGTCATGATAATAATGGTTTCTTAGACGTCCTAGGCATGCgatccaatatcaaaggaaatg-3'

MQ1p: Fwd, PCR amplify $2 \mu$ locus, mediate insertion into Aatll-digested pDN61x family vectors to create pDN62x family vectors.

5'ATAATGTATGCTATACGAAGTTATTGACGTcaacgaagcatctgtgcttcattttg-3'

MQ2p: Rev, PCR amplify $2 \mu$ locus, mediate insertion into Aatll-digested pDN61x family vectors to create $\mathrm{pDN} 62 \mathrm{x}$ family vectors.

5'TATAGCATACATTATACGAAGTTATGACGTcgatccaatatcaaaggaaatgatagc-3'

DN661p: Sequencing primer for yeast replication loci, anneals near yeast selectable marker locus.

5'tacaatctgctctgatgcc-3'

DN837p: Sequencing primer for yeast replication loci, anneals in AmpR promoter. 5'ttattgaagcatttatcaggg-3' 
DN680p: Rev, PCR amplify 200 bases of CPS1 terminator to copy CPYpr::GFP-CPS1 cassette, mediate repair of Pvull-digested pDN616.

5'GATCGGTGCGGGCCTCTTCGCTATTACGCCAGtaaattttgatttgacacttg-3'

DN693p: Fwd, PCR amplify 455 bases of PRC1 promoter to copy PRC1pr::GFP-CPS1 cassette, mediate repair of Pvull-digested pDN616.

5'CCTCTCCCCGCGCGTTGGCCGATTCATTAATGCAGattgacagagcagtatgtgagg-3'

DN927p: Fwd, PCR amplify 500 bp SEC17 promoter, mediate gap repair into Sacl-digested pDN516.

5'CTAGTTCTAGAGCGGCCGCCACCGCGGTGGAGCTCttctttgtcaattgcatctcta-3'

DN928p: Rev, PCR amplify 300 bp of SEC17 terminator, mediate gap repair into Sacl-digested pDN5xx.

5'TAACCCTCACTAAAGGGAACAAAAGCTGGAGCTCggaagatccttacattacacg-3'

DN982p: Fwd, introduce L291A L292A ('LALA') mutation into SEC17 via sequence overlap extension PCR, overlaps with DN983p.

5'ATCCAGCAACAAGAAGATGAT GCG GCA TGA acggcatatacttacgcgca-3'

DN983p: Rev, introduce L291A L292A ('LALA') mutation into SEC17 via sequence overlap extension PCR, overlaps with DN982p.

5'TGCGCGTAAGTATATGCCGT TCA TGC CGC atcatcttcttgttgctggat-3' 
Nickerson et al. p20

\section{FIGURES \& LEGENDS}

Figure 1
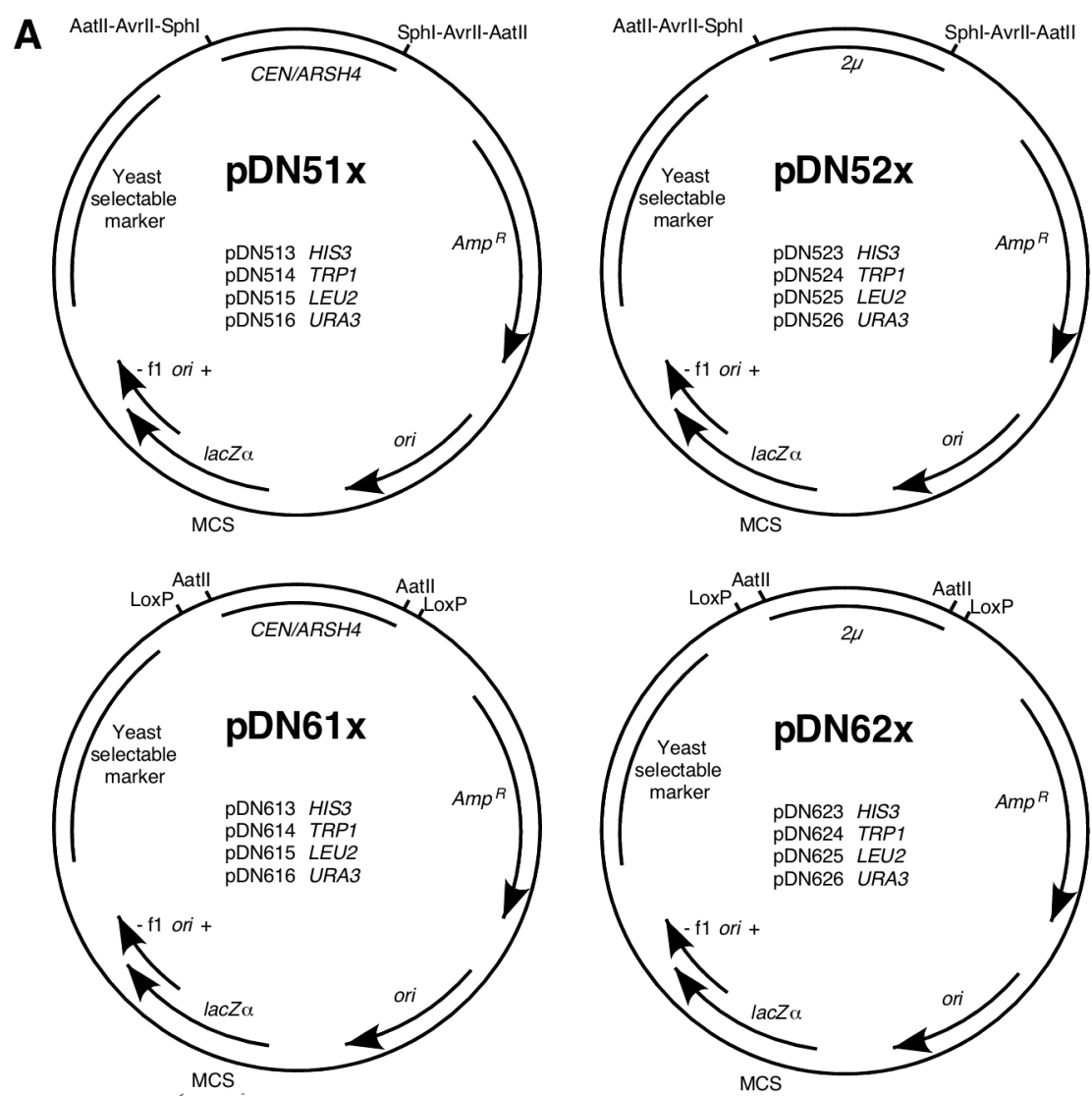

B
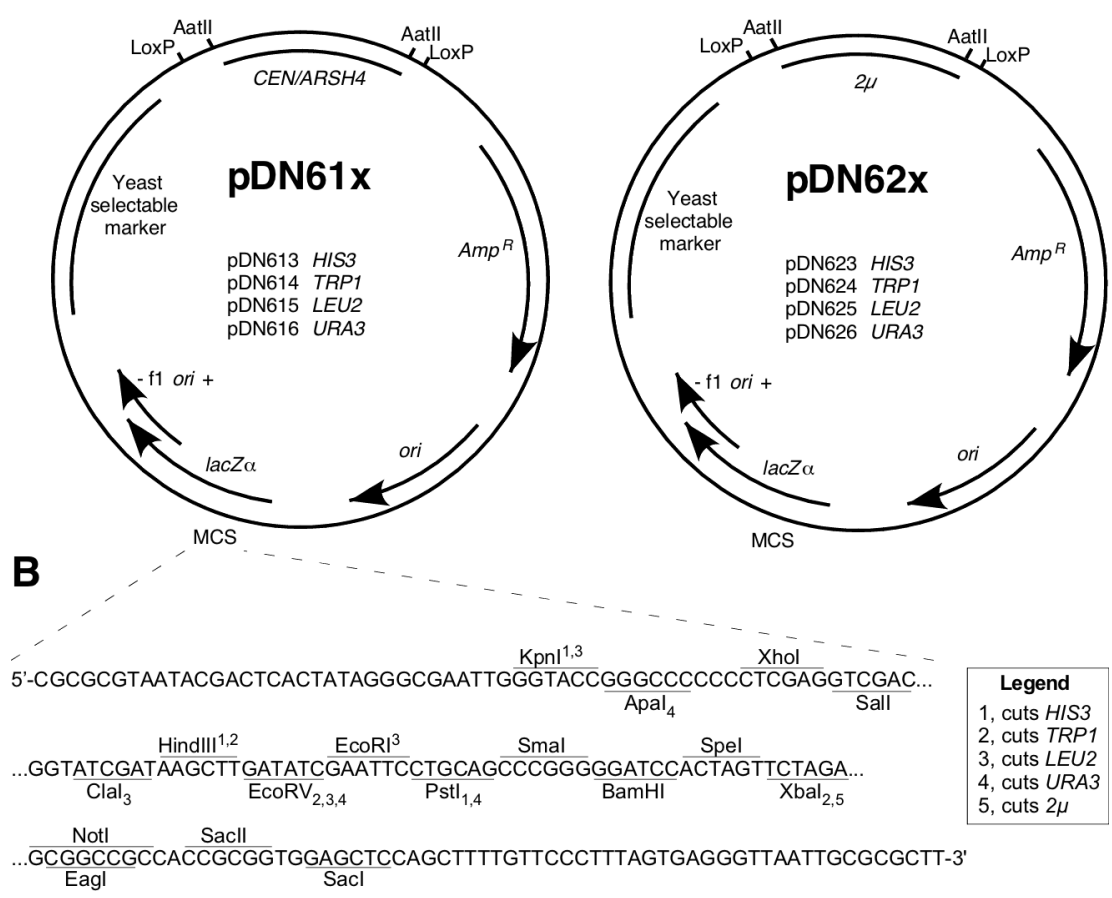

Figure 1. Functional maps for pDN5xx and pDN6xx series of low-copy and high-copy vectors. A) Maps displaying consistent architectural features and specific functional differences of pDN5xx and pDN6xx families. Selected restriction enzyme cut sites and LoxP sequences flanking replication loci are displayed. B) Multiple cloning site (MCS) in focus, displaying nucleotide sequence of single strand (template strand for $\operatorname{lac} Z \alpha, \beta$-galactosidase) and unique restriction enzyme cut sites. Subscript and superscript numerals with each enzyme indicate capacity for enzyme to cut yeast selectable marker loci as indicated in legend. CEN, centromeric sequence, low copy yeast replication locus. $A R S H 4$, autonomous replicating sequence. $2 \mu$, yeast high copy replication locus. $A m p^{R}$, ampicillin resistance gene ( $\beta$-lactamase). ori, high copy $E$. coli origin or replication. f1 ori, f1 bacteriophage origin of replication. Plasmid loci depicted at approximate scale. Full plasmid sequences and annotated maps are available in Supplementary Materials. 
Nickerson et al. p21

\section{Figure 2}

A

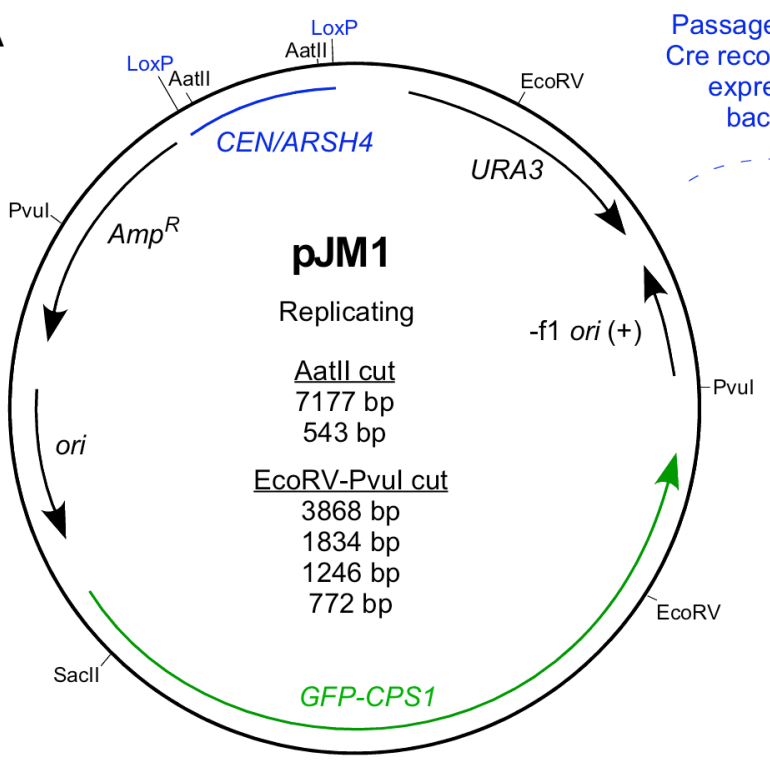

B

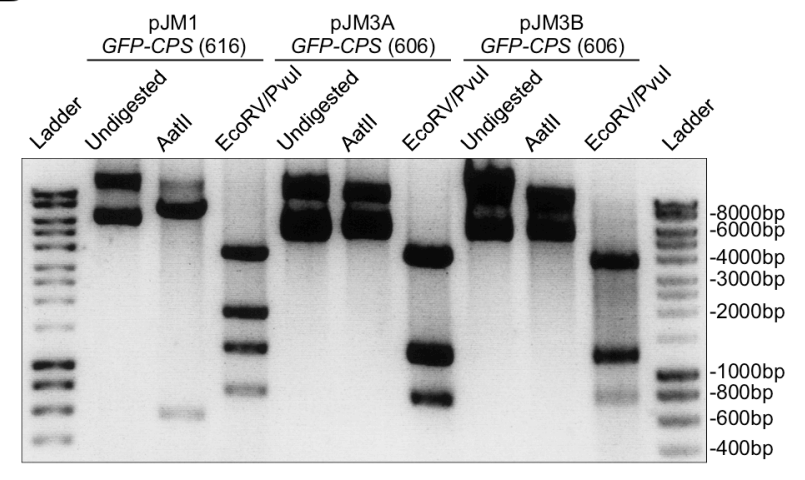

Passage through re recombinaseexpressing bacteria

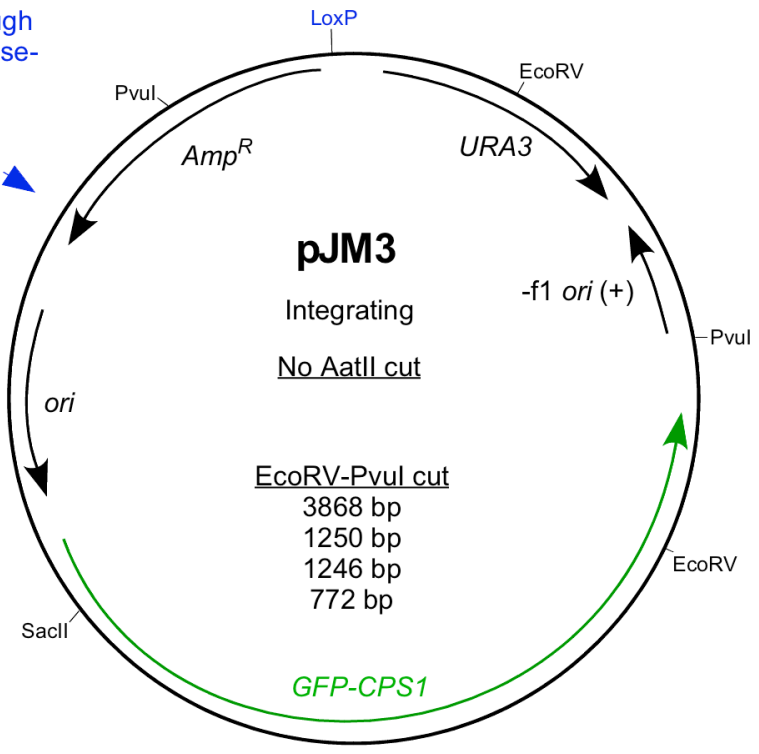

C

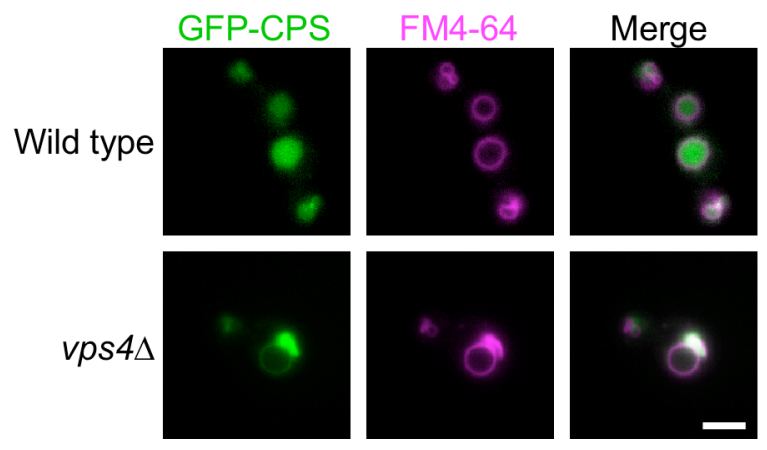

Figure 2. Conversion of replicating, episomal vector to integrating vector via Cre recombinase. A) Plasmid maps of low copy replicating (pJM1) and integrating (pJM3) plasmids, including relevant endonuclease enzyme cut sites and predicted restriction fragment product sizes. B) Agarose gel electrophoretic analysis of restriction digest products derived from pre-Cre-treated plasmid pJM1 and post-Cre-treated plasmid pJM3 candidates A and B. Note that $1246 \mathrm{bp}$ and $1250 \mathrm{bp}$ fragments predicted from EcoRV-Pvul double digests of pJM3 appear as a single band. Undigested and uncut plasmids show high molecular weight bands representing supercoiled, nicked, and concatenated circular DNA whose gel migration should not be compared to linear ladder size standards. Unlabeled DNA ladder bands are of length halfway between neighboring labeled bands. C) Fluorescence microscopy of FM 4-64-labeled, logarithmic phase yeast expressing chromosomally integrated pJM3. pJM3 was digested with Sacll to integrate in the chromosomal PCR1 promoter. Chromosomal integrants were selected on media lacking uracil. Cells were cultured in non-selective media prior to imaging. Scale bar $=1 \mu \mathrm{m}$. 
Nickerson et al. p22

\section{Figure 3}
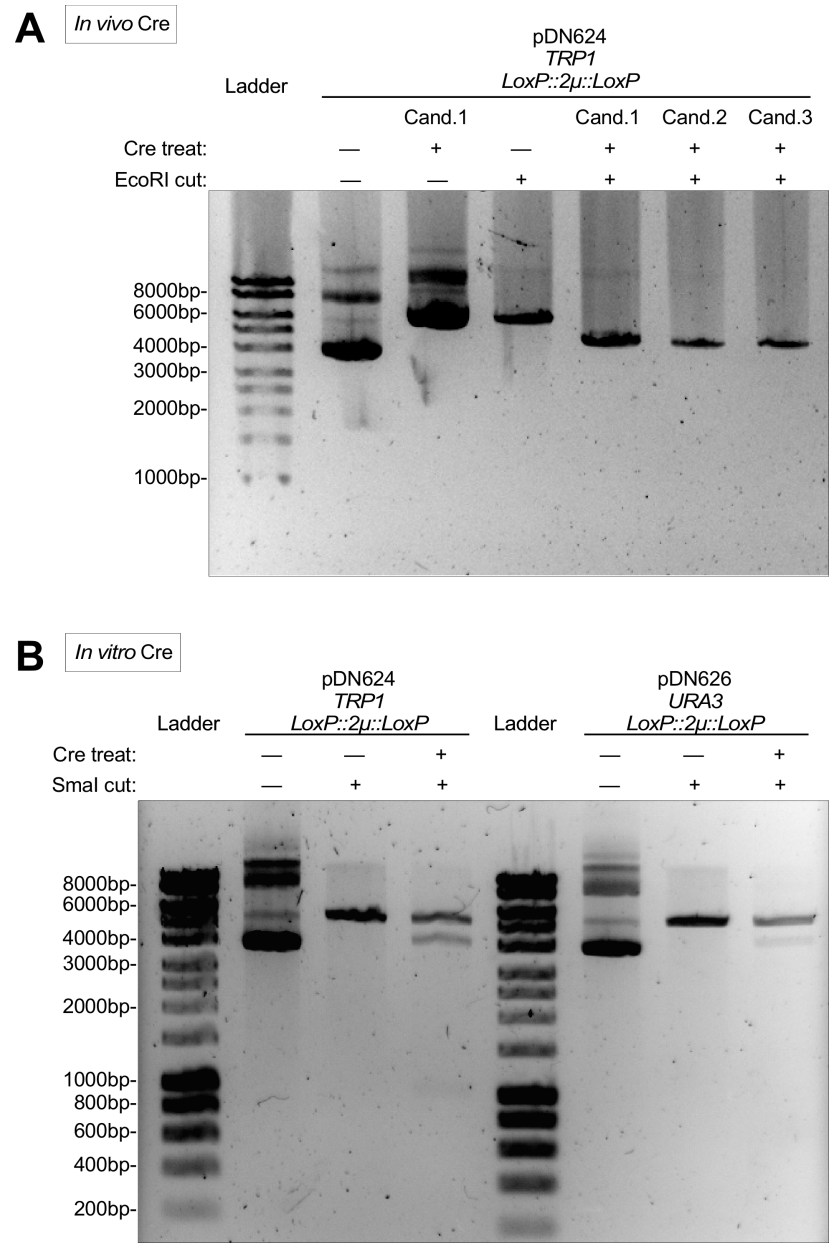

Figure 3. Cre-mediated removal of $2 \mu$ replication locus in vivo and in vitro. A) Agarose gel electrophoretic confirmation of removal of $2 \mu$ replication locus after passage of pDN624 through Cre-expressing bacterial strain N2114Sm. Each candidate represents a unique plasmid isolate from a single N2114Sm pDN624 colony. EcoRI-cut (linearized) pDN624 produces predicted bands of 5692 bp and 4302 bp before and after removal of $2 \mu$ locus, respectively. B) Agarose gel electrophoretic confirmation of removal of $2 \mu$ replication loci after in vitro treatment of pDN624 and pDN626 with Cre recombinase. Cre-treated samples represent polyclonal populations that include both unmodified (5692 bp for pDN624; 5802 bp for pDN626) and modified plasmids (4302 for pDN624; 4412 for pDN626). Unlabeled DNA ladder bands are of length halfway between neighboring labeled bands. 
Nickerson et al. p23

\section{Figure 4}

A

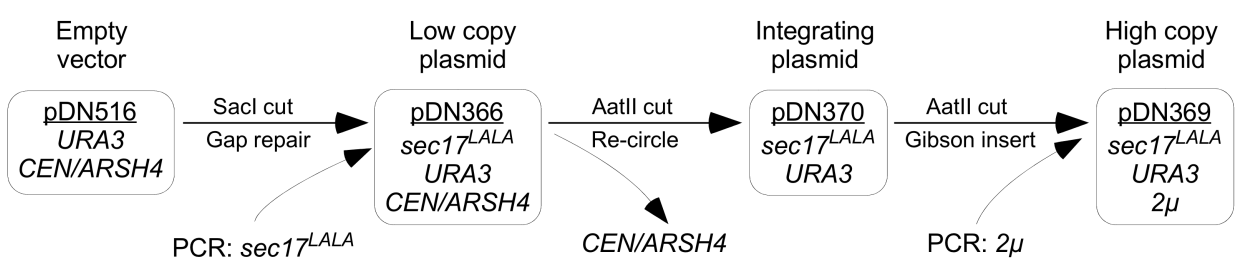

B

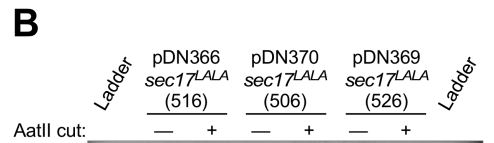

C

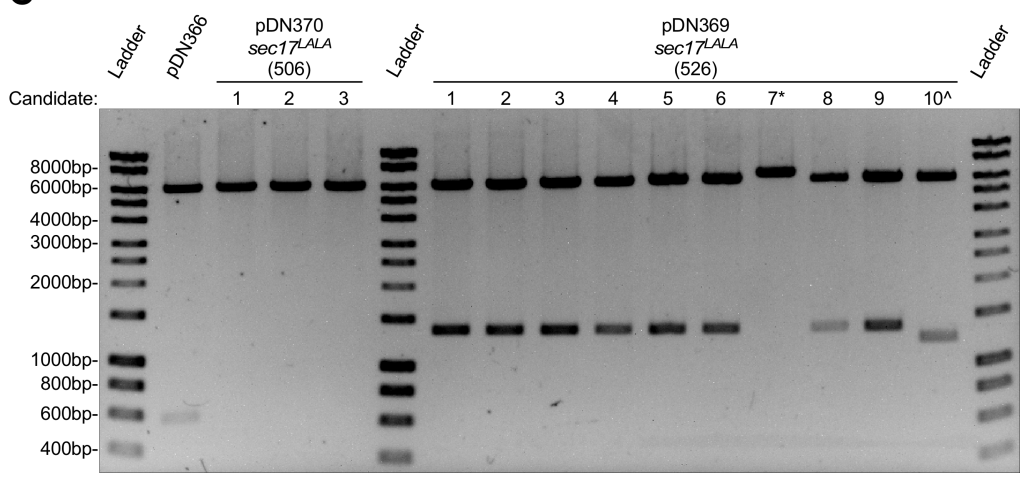

Figure 4. Example workflow to generate low copy, high copy, and integrating plasmids from a common precursor. A) Workflow schematic representing modification of original low copy replicating vector by insertion of a PCR product at MCS, followed by removal of original replication locus and replacement with high copy replication locus. B) Agarose gel electrophoresis and restriction enzyme analysis of plasmids resulting from demonstrated workflow. Observed Aatll restriction fragments conform to predicted sizes: $6185 \mathrm{bp}$ and $563 \mathrm{bp}$ for pDN366; 6185 bp for pDN370; and 6185 bp and 1369 bp for pDN369. C) Agarose gel electrophoretic analysis of efficiency of removal of CEN/ARSH4 replication locus and replacement with $2 \mu$ replication locus. All samples shown were digested with Aatll to linearize vector (no replication locus) or cut on either side of replication locus. ${ }^{*}$ and ${ }^{\wedge}$ symbols indicate failed pDN369 candidates. Unlabeled DNA ladder bands are of length halfway between neighboring labeled bands. 
Nickerson et al. p24

\section{REFERENCES}

Agaphonov, M., and A. Alexandrov, 2014 Self-excising integrative yeast plasmid vectors containing an intronated recombinase gene. FEMS Yeast Research 14: 1048-1054.

Babst, M., T. K. Sato, L. M. Banta, and S. D. Emr, 1997 Endosomal transport function in yeast requires a novel AAA-type ATPase, Vps4p. The EMBO Journal 16: 1820-1831.

Botstein, D., and G. R. Fink, 2011 Yeast: An Experimental Organism for 21st Century Biology. Genetics 189: 695-704.

Carter, Z., and D. Delneri, 2010 New generation of loxP-mutated deletion cassettes for the genetic manipulation of yeast natural isolates. Yeast 27: 765-775.

Chee, M. K., and S. B. Haase, 2012 New and Redesigned pRS Plasmid Shuttle Vectors for Genetic Manipulation of Saccharomyces cerevisiae. G3: Genes, Genomes, Genetics 2: 515-526.

Christianson, T. W., R. S. Sikorski, M. Dante, J. H. Shero, and P. Hieter, 1992 Multifunctional yeast high-copy-number shuttle vectors. Gene 110: 119-122.

Da Silva, N. A., and S. Srikrishnan, 2012 Introduction and expression of genes for metabolic engineering applications in Saccharomyces cerevisiae. FEMS Yeast Res 12: 197-214.

Fang, F., K. Salmon, M. W. Y. Shen, K. A. Aeling, E. Ito et al., 2011 A vector set for systematic metabolic engineering in Saccharomyces cerevisiae. Yeast 28: 123-136.

Futcher, B., and J. Carbon, 1986 Toxic effects of excess cloned centromeres. Molecular and Cellular Biology 6: 2213-2222.

Gibson, D. G., 2009 Synthesis of DNA fragments in yeast by one-step assembly of overlapping oligonucleotides. Nucleic Acids Res 37: 6984-6990. 
Gibson, D. G., L. Young, R.-Y. Chuang, J. C. Venter, C. A. Hutchison lii et al., 2009 Enzymatic assembly of DNA molecules up to several hundred kilobases. Nature Methods 6: 343345.

Gietz, D., A. S. Jean, R. A. Woods, and R. H. Schiestl, 1992 Improved method for high efficiency transformation of intact yeast cells. Nucleic Acids Res 20: 1425-1425.

Gueldener, U., J. Heinisch, G. J. Koehler, D. Voss, and J. H. Hegemann, 2002 A second set of loxP marker cassettes for Cre-mediated multiple gene knockouts in budding yeast. Nucleic Acids Res 30: e23-e23.

Guthrie, C., and G. R. Fink, 2002 Guide to yeast genetics and molecular biology. Academic Press, San Diego, CA.

Jensen, N. B., T. Strucko, K. R. Kildegaard, F. David, J. Maury et al., 2014 EasyClone: method for iterative chromosomal integration of multiple genes Saccharomyces cerevisiae. FEMS Yeast Res 14: 238-248.

Lee, M. E., W. C. DeLoache, B. Cervantes, and J. E. Dueber, 2015 A Highly Characterized Yeast Toolkit for Modular, Multipart Assembly. ACS Synth. Biol. 4: 975-986.

Lobingier, B. T., D. P. Nickerson, S.-Y. Lo, and A. J. Merz, 2014 SM proteins Sly1 and Vps33 coassemble with Sec17 and SNARE complexes to oppose SNARE disassembly by Sec18 (R. Jahn, Ed.). eLife 3: e02272.

Mead, D. J., D. C. J. Gardner, and S. G. Oliver, 1986 The yeast $2 \mu$ plasmid: strategies for the survival of a selfish DNA. Mol Gen Genet 205: 417-421.

Nickerson, D. P., and A. J. Merz, 2015 LUCID: A Quantitative Assay of ESCRT-Mediated Cargo Sorting into Multivesicular Bodies. Traffic 16: 1318-1329. 
Nickerson, D. P., M. R. G. Russell, S.-Y. Lo, H. C. Chapin, J. M. Milnes et al., 2012 Termination of Isoform-Selective Vps21/Rab5 Signaling at Endolysosomal Organelles by Msb3/Gyp3. Traffic 13: 1411-1428.

Odorizzi, G., M. Babst, and S. D. Emr, 1998 Fab1p PtdIns(3)P 5-Kinase Function Essential for Protein Sorting in the Multivesicular Body. Cell 95: 847-858.

Paulsel, A. L., A. J. Merz, and D. P. Nickerson, 2013 Vps9 family protein Muk1 is the second Rab5 guanosine nucleotide exchange factor in budding yeast. J. Biol. Chem. jbc.M113.457069.

Resnick, M. A., J. Westmoreland, and K. Bloom, 1990 Heterogeneity and maintenance of centromere plasmid copy number inSaccharomyces cerevisiae. Chromosoma 99: 281288.

Robinson, J. S., D. J. Klionsky, L. M. Banta, and S. D. Emr, 1988 Protein sorting in Saccharomyces cerevisiae: isolation of mutants defective in the delivery and processing of multiple vacuolar hydrolases. Molecular and Cellular Biology 8: 4936-4948.

Rose, M. D., F. Winston, and P. Hieter, 1990 Methods in Yeast Genetics: A Laboratory Course Manual. Cold Spring Harbor Laboratory Press, Cold Spring Harbor, NY.

Schwartz, M. L., D. P. Nickerson, B. T. Lobingier, R. L. Plemel, M. Duan et al., 2017 Sec17 ( $\alpha-$ SNAP) and an SM-tethering complex regulate the outcome of SNARE zippering in vitro and in vivo (A. T. Brunger, Ed.). eLife 6: e27396.

Seifert, H. S., E. Y. Chen, M. So, and F. Heffron, 1986 Shuttle mutagenesis: a method of transposon mutagenesis for Saccharomyces cerevisiae. PNAS 83: 735-739. 
Nickerson et al. p27

Sherman, F., 2002 Getting started with yeast, pp. 3-41 in Methods in Enzymology, edited by C. Guthrie and G. R. Fink. Guide to Yeast Genetics and Molecular and Cell Biology - Part B, Academic Press.

Shideler, T., D. P. Nickerson, A. J. Merz, and G. Odorizzi, 2015 Ubiquitin binding by the CUE domain promotes endosomal localization of the Rab5 GEF Vps9. MBoC 26: 1345-1356.

Siddiqui, M. S., A. Choksi, and C. D. Smolke, 2014 A system for multilocus chromosomal integration and transformation-free selection marker rescue. FEMS Yeast Research 14: 1171-1185.

Sikorski, R. S., and P. Hieter, 1989 A system of shuttle vectors and yeast host strains designed for efficient manipulation of DNA in Saccharomyces cerevisiae. Genetics 122: 19-27.

Vida, T. A., and S. D. Emr, 1995 A new vital stain for visualizing vacuolar membrane dynamics and endocytosis in yeast. J Cell Biol 128: 779-792. 